\title{
Tyrosine 450 in the Voltage- and Calcium-Gated Potassium Channel of Large Conductance Channel Pore-Forming (slo1) Subunit Mediates Cholesterol Protection against Alcohol-Induced Constriction of Cerebral Arteries ${ }^{\llbracket}$
}

\author{
Kelsey North, Shivantika Bisen, Alex M. Dopico, and Anna N. Bukiya \\ Department of Pharmacology, College of Medicine, University of Tennessee Health Science Center, Memphis, Tennessee
}

Received May 8, 2018; accepted August 15, 2018

\begin{abstract}
Alcohol (ethanol) at physiologically relevant concentrations $(<100 \mathrm{mM})$ constricts cerebral arteries via inhibition of voltage- and calcium-gated potassium channels of large conductance (BK) located in vascular smooth muscle (VSM). These channels consist of channel-forming slo1 (cbv1, KCNMA1) and accessory beta1 (KCNMB1) subunits. An increase in VSM cholesterol (CLR) via either dietary CLR intake or in vitro CLR enrichment was shown to protect against endothelium-independent, alcohol-induced constriction of cerebral arteries. The molecular mechanism(s) of this protection remains unknown. Here, we demonstrate that CLR enrichment of de-endothelialized middle cerebral arteries (MCAs) of rat increased CLR content in the VSM in a concentration-dependent manner. CLR enrichment blunted MCA constriction evoked by $18-75 \mathrm{mM}$ but not by $100 \mathrm{mM}$ alcohol. MCA enrichment with coprostanol (COPR) also blunted
\end{abstract}

vasoconstriction by $50 \mathrm{mM}$ alcohol, despite the fact that COPR and CLR differ in their ability to modify several major physical properties of the bilayer. CLR protection against 50 but not $100 \mathrm{mM}$ alcohol was also observed in C57BL/6 and KCNMB1 knockout (KO) mice. Permeabilization of KCNMA1 KO MCAs with Y450Fcbv1 totally ablated CLR, but not COPR protection against vasoconstriction by $50 \mathrm{mM}$ alcohol. Thus, CLR and alcohol interact at the level of the BK channel slo1 subunit, with Y450 being critical for CLR protection against alcohol-induced vasoconstriction. We document for the first time a functional competition between CLR and alcohol in regulating cerebral artery diameter and a critical role of a single amino acid within the BK channel pore-forming subunit in controlling CLR-alcohol interaction at the organ level.

\section{Introduction}

Cholesterol (CLR) is a major lipid constituent of mammalian cell membranes (Veerkamp, 1972; Dopico and Tigyi, 2007) and determines many membrane physical properties (Heiner et al., 2008; Haldar et al., 2012). At the organismal level, CLR serves as a precursor of steroid hormones and as a critical signaling molecule that plays a major role in cardio/ cerebrovascular physiology (Miller and Auchus, 2011). Pathologic conditions that arise from either a lack or abundance of CLR range from neurodevelopmental delay to severe vascular disorders (Martín et al., 2014; Ivanovic and Tadic, 2015). Hypercholesterolemia has widespread effects on vascular function via cholesterol-driven changes in membrane fluidity, enzyme activity, and cation transporter function in endothelial cells, cardiomyocytes, and vascular smooth muscle (VSM) cells (Li et al., 2014; Wu et al., 2015). Thus, it is not surprising that

This work was supported by the National Institutes of Health National Institute of Alcohol Abuse and Alcoholism [Grant R01 AA023764 to A.N.B.; Grant R37 AA11560 to A.M.D.].

https://doi.org/10.1124/jpet.118.250514.

S This article has supplemental material available at jpet.aspetjournals.org. hypercholesterolemia constitutes a risk factor for vascular, including cerebrovascular, diseases (van Rooy and Pretorius, 2014).

Independent of any other factor, moderate-to-heavy episodic alcohol intake, such as binge drinking, is associated with an increased risk for cerebrovascular spasm and death from stroke (Zakhari, 1997; Zhang et al., 2014). Cerebrovascular disease associated with moderate-to-heavy alcohol intake is independent of beverage type and alcohol metabolism in the body, but is linked to the pharmacological actions of ethanol (ethyl alcohol) itself (Altura and Altura, 1984). Moreover, studies in humans and laboratory animal species document that acute alcohol administration at concentrations equivalent to blood alcohol levels that are above the legal limit of intoxication $(>18 \mathrm{mM})$ constricts cerebral arteries both in vitro and in vivo (Altura and Altura, 1984; Bukiya et al., 2014).

Alcohol-induced constriction of cerebral arteries is endothelium independent and enabled by alcohol inhibition of voltageand calcium-gated potassium channels of large conductance (BK) in VSMs (Liu et al., 2004). Functional BK channels are conformed by a tetramer of channel-forming alpha subunits (slo1 proteins, encoded by KCNMA1) (Salkoff et al., 2006).

ABBREVIATIONS: BK, voltage- and calcium-gated potassium channel of large conductance; CLR, cholesterol; COPR, coprostanol; CRAC, cholesterol recognition amino acid consensus; $\mathrm{EtOH}$, ethanol; $\mathrm{KO}$, knockout; $\mathrm{M} \beta \mathrm{CD}$, methyl-beta-cyclodextrin; MCA, middle cerebral artery; PSS, physiologic sodium saline; VSM, vascular smooth muscle; WT, wild type. 
However, in smooth muscle cells (including vascular myocytes) slo1 subunits are accompanied by accessory beta1 proteins (Brenner et al., 2000b; Orio et al., 2002). The latter modify the BK current phenotype and pharmacological profile (Brenner et al., 2000a; Bukiya et al., 2007, 2009b). Moreover, deletion of the beta1-coding KCNMB1 gene in mouse diminishes alcoholinduced constriction of cerebral arteries (Bukiya et al., 2009a).

In addition to subunit composition, BK channel sensitivity to alcohol is tuned by CLR levels in VSM. Indeed, we have recently shown that a high-CLR diet resulted in elevated CLR level within the cerebral artery smooth muscle; this elevation being protective against cerebral artery constriction evoked by $50 \mathrm{mM}$ alcohol in vivo and in vitro (Bukiya et al., 2014). Notably, CLR protection against alcohol-induced constriction of the cerebral artery remained unaffected by the removal of functional endothelium, and thus represented an endotheliumindependent phenomenon (Bukiya et al., 2014). Protection against alcohol-induced constriction was observed not only after CLR accumulation in the VSM by high-CLR dietary intake in vivo, but also following in vitro CLR enrichment using the steroid carriers methyl-beta-cyclodextrin or lowdensity lipoprotein (Bukiya et al., 2014; Bisen et al., 2016). On the other hand, high-CLR diet-driven protection against alcohol-induced constriction of cerebral arteries was removed upon in vitro depletion of excessive CLR from de-endothelialized cerebral vessels (Bukiya et al., 2014).

Previous studies underscored that a CLR antagonism of alcohol effect could be observed even in the absence of the BK beta1 subunit (Bisen et al., 2016) and in a very simple environment of artificial lipid bilayer (Crowley et al., 2003). These findings raise the hypothesis that the slo1 protein itself is the molecular effector of such antagonism. Slo1 subunits contain 10 cholesterol recognition amino acid consensus (CRAC) motifs. Mutations within the membrane-adjacent CRAC4 led to partial removal of BK channel CLR sensitivity (Singh et al., 2012). Thus, we hypothesize that CRAC4 plays a major role in the CLR-alcohol interaction and its impact on cerebral artery diameter.

In the current study, we used Sprague-Dawley rats and KCNMB1 and KCNMA1 knockout (KO) mice to evaluate whether CLR was able to protect against alcohol-induced constriction of cerebral arteries when alcohol was probed at a toxicology relevant range [18-100 $\mathrm{mM}$ ethanol (EtOH)], and to begin to address the molecular determinants of CLR-alcohol interaction in the control of cerebral artery diameter at the smooth muscle level. Alcohol concentrations chosen span from the legal limit of intoxication for driving a motor vehicle within the majority of the United States $(18 \mathrm{mM})$ and blood alcohol content detected after moderate-to-heavy alcohol intake (35-75 mM), up to levels that may be lethal to humans $(100 \mathrm{mM})$ (Heatley and Crane, 1990).

\section{Materials and Methods}

Ethical Aspects of Research. The care of animals and experimental protocols were reviewed and approved by the Animal Care and Use Committee of the University of Tennessee Health Science Center, which is an institution accredited by the Association for Assessment and Accreditation of Laboratory Animal Care International. To avoid the extensive use of animal donors, most of the experiments were performed on rat cerebral arteries, which are larger and easier to handle when compared with their mouse counterparts. However, a KO mouse model was also introduced when its use was justified by experimental needs.

Cerebral Artery Diameter Measurement. Adult male Sprague-Dawley rats $(\approx 250 \mathrm{~g})$ and 8 - to 12 -week-old male C57BL/6 control, KCNMB1 KO, or KCNMA1 KO mice were deeply anesthetized with isoflurane via inhalation. Resistance-size middle cerebral arteries (MCAs) were dissected out of the rat or mouse brain, following animal euthanasia with a guillotine or sharp scissors, respectively. The endothelium was removed by passing an air bubble into the vessel lumen for 90 seconds immediately prior to vessel cannulation. This method has been consistently used by our group, and validated using endothelium-dependent versus endothelium-independent vasodilators (Bukiya et al., 2007, 2011). Arteries were cannulated as previously described by our group (Bukiya et al., 2011, 2013). Briefly, MCAs were cut into 5-10-mm-long segments under a microscope (SMZ645, Nikon, Tokyo, Japan). The segment was cannulated at each end, and the artery exterior was continuously perfused with physiologic sodium saline (PSS) of the following composition: $119 \mathrm{mM} \mathrm{NaCl}, 4.7 \mathrm{mM} \mathrm{KCl}, 1.2 \mathrm{mM} \mathrm{KH}_{2} \mathrm{PO}_{4}, 1.6 \mathrm{mM}$ $\mathrm{CaCl}_{2}, 1.2 \mathrm{mM} \mathrm{MgSO}$, $0.023 \mathrm{mM}$ EDTA, $11 \mathrm{mM}$ glucose, and $24 \mathrm{mM}$ $\mathrm{NaHCO}_{3}(\mathrm{pH}=7.4)$. PSS was continuously bubbled with $\mathrm{O}_{2} / \mathrm{CO}_{2} / \mathrm{N}_{2}$ $(21 \% / 5 \% / 74 \%)$ gas mixture and maintained at $35-37^{\circ} \mathrm{C}$. The artery external wall diameter was measured using the automatic edgedetection function of the IonWizard software (IonOptix, Westwood, MA) via a Sanyo VCB-3512T camera (Sanyo Electric Co., Moriguchi, Japan). Arteries were first incubated at an intravascular pressure of $10 \mathrm{~mm} \mathrm{Hg}$ for 10 minutes. Then, intravascular pressure was increased to $60 \mathrm{~mm} \mathrm{Hg}$ and held steady throughout the experiment to induce myogenic tone development and maintenance. Each artery segment was only exposed to ethanol once to avoid development of desensitization during repeated applications of ethanol-containing solution (Bukiya et al., 2011). At the end of each experiment, the artery was probed with $\mathrm{Ca}^{2+}$-free PSS to verify the viability of the artery segment. If the artery failed to dilate in $\mathrm{Ca}^{2+}$-free solution, data from this artery segment were excluded from further analysis. When required by experimental design, arterial contractility was probed with a high-KCl solution of the following composition: $63.7 \mathrm{mM} \mathrm{NaCl}, 60 \mathrm{mM} \mathrm{KCl}, 1.2 \mathrm{mM} \mathrm{KH}_{2} \mathrm{PO}_{4}, 1.2 \mathrm{mM} \mathrm{MgSO}$, $0.023 \mathrm{mM}$ EDTA, $11 \mathrm{mM}$ glucose, $24 \mathrm{mM} \mathrm{NaHCO}$, and $1.6 \mathrm{mM}$ $\mathrm{CaCl}_{2}$.

Cholesterol and Coprostanol Enrichment of the Arteries. For steroid enrichment, we followed previously described methodology (Zidovetzki and Levitan, 2007; Bukiya et al., 2011). For rat and mouse cerebral artery diameter measurements and immunofluorescence experiments, arteries were subjected to a 1-hour-long incubation in PSS containing $5 \mathrm{mM}$ methyl-beta-cyclodextrin $(\mathrm{M} \beta \mathrm{CD}): 0.625 \mathrm{mM}$ steroid complex (8:1 molar ratio). To ensure $\mathrm{M} \beta \mathrm{CD}$ saturation with $\mathrm{CLR}$, the solution was shaken at $37^{\circ} \mathrm{C}$ overnight and filtered prior to the artery incubation. Steroid enrichment of the arteries was performed immediately prior to artery cannulation for diameter measurements or for immunofluorescence staining.

For filipin staining, serial dilutions of the $\mathrm{M} \beta \mathrm{CD}$ :steroid complex were performed from the starting $50 \mathrm{mM} \mathrm{M} \beta \mathrm{CD}: 6.25 \mathrm{mM}$ steroid complex. Arteries were subjected to a 1-hour-long incubation in PSS containing the various concentrations of $\mathrm{M} \beta \mathrm{CD}$ :steroid as outlined in Results.

Fluorescence Staining of Steroid Content in the Artery Wall Using Filipin. Arteries were fixed in $4 \%$ paraformaldehyde for 30 minutes in the dark at room temperature. Permeabilization was performed using $0.5 \%$ Triton-100 in phosphate-buffered saline for 10 minutes. Following washout, arteries were stained with $25 \mu \mathrm{g} / \mathrm{ml}$ filipin solution for 1 hour in the dark. Filipin solution was prepared by diluting $10 \mathrm{mg} / \mathrm{ml}$ filipin stock in dimethylsulfoxide, which was stored at $-20^{\circ} \mathrm{C}$ for no longer than 1 week. After washing, arteries were mounted onto glass slides using ProLong Gold antifade reagent (Invitrogen, Eugene, OR), dried at room temperature in the dark for 24 hours, and sealed with clear nail polish. Sealed slides were stored at $-20^{\circ} \mathrm{C}$. 
Biochemical Determination of CLR Level in the Artery Tissue. For the time course of CLR enrichment, arteries were subjected to incubation with $5 \mathrm{mM} \mathrm{M} \beta \mathrm{CD}: 0.625 \mathrm{mM}$ CLR complex in PSS for various time intervals $(5,10,20,30$, and 60 minutes). Artery tissue was homogenized as previously described (Bukiya et al., 2011). Protein and CLR levels were determined using the Pierce BCA Protein Assay Kit (Thermo Scientific, Waltham, MA) and Amplex Red Cholesterol Assay Kit (Molecular Probes, Inc., Eugene, OR), respectively. Protein and CLR readings were performed with a microplate reader (Synergy, New York City, NY) using absorbance (562 nm) and fluorescence (excitation/ emission $=540 / 590 \mathrm{~nm}$ ) reading functions, respectively.

Fluorescence Data Acquisition. Immunofluorescence images were obtained using the $405 \mathrm{~nm}$ laser line of an Olympus FV-1000 laser scanning confocal system (Olympus, Center Valley, PA). Imaging was performed using a z-stack function on the microscope, with the thickness of the step set at $1 \mu \mathrm{m}$. Stacks also contained recordings of the images obtained within the visible light spectrum. Within each artery, three to four areas were randomly picked; within each area, the total fluorescence signal was measured to represent a single data point.

KCNMA1 KO Mouse Artery Permeabilization. KCNMA1 (slo1-lacking) KO mouse MCAs were dissected out and permeabilized to allow delivery of pcDNA3 vector containing slo1 subunit cloned from rat cerebral artery (cbv1; AAP82453) with mutated CRAC4 domain (Y450F cbv1) (Singh et al., 2012). pcDNA3 vector without gene insert and with wild-type (WT) cbv1 was used as the negative and positive controls, respectively. Permeabilization was performed following a standard chemical loading/reverse permeabilization technique (Lesh et al., 1995; Xi et al., 2008).

Computational Chemical Modeling. A protein homology model of the cbv1 (GenBank AAP82453) cytosolic tail domain was created using the built-in function in the Molecular Operating Environment 2008 software program (Chemical Computing Group, Montreal, Canada). The crystal structure of human slo1 (Protein Data Bank ID 3MT5) was used as a template. CLR and coprostanol (COPR) chemical conformation libraries were created and docked onto CRAC4-forming amino acids V444, Y450, and K353 using default settings of the built-in conformational library and docking suit in Molecular Operating Environment.

Chemicals. CLR was purchased from Avanti Polar Lipids (Alabaster, AL). Ethanol (200 proof; ultra-pure) was purchased from American Bioanalytical (Natick, MA). All other chemicals were purchased from Sigma Aldrich (St. Louis, MO). Ethanol was freshly diluted in PSS solution immediately before application to the artery. Each pressurized artery was exposed to ethanol only once to avoid reduced responsiveness to multiple applications of ethanol (Bukiya et al., 2011).

Data Analysis. Artery diameter data were analyzed using the IonWizard 4.4 software program (IonOptix). The value for arterial diameter before drug application was obtained by averaging diameter values from the same arterial segment during 3 minutes of recording immediately before drug application. A drug-induced change in arterial diameter was determined from the peak effect obtained during drug application.

Statistical analysis was performed using the InStat 3.05 software program (GraphPad, San Diego, CA). When the number of observations in the groups under comparison exceeded 6, and the Gaussian distribution of the data was confirmed by the Kolmogorov-Smirnov test, analysis was performed using the unpaired Student's $t$ test. In both cases, statistical analysis was conducted using the MannWhitney nonparametric test. In all cases, testing assumed two-tail $P$ values. For comparison of multiple experimental groups, the Kruskal-Wallis test and Dunn's post-test were used. In all cases, significance was set at $P<0.05$. Data were expressed as mean \pm S.E.M.; $n=$ number of arteries. In each experimental group, individual arteries were obtained from different animal donors. Final plotting and fitting of data were conducted using the Origin 8.5 software program (Origin Laboratory, Northampton, MA).

\section{Results}

Cholesterol Modulation of Alcohol Action on Cerebral Artery Diameter Appears to be Competitive. To determine the alcohol concentration range at which CLR protects against alcohol-induced constriction of cerebral arteries, we dissected MCAs out of rat brains. For CLR enrichment, each artery was incubated in $\mathrm{M} \beta \mathrm{CD}$ :CLR complex prior to cannulation, while a control group of arteries with naive CLR was incubated in PSS solution for 1 hour. To verify the efficiency of the CLR enrichment procedure, we stained the arteries with filipin, a cholesterol-sensitive dye (Muller et al., 1984). Arteries were incubated at room temperature for 1 hour in $\mathrm{M} \beta \mathrm{CD}$ :CLR complex containing increasing concentrations of CLR (from 0.00625 to $6.25 \mathrm{mM}$ ).

The cerebral artery myocyte layer within tunica media was identified by a positive immunofluorescence staining against BK channel smooth muscle-specific beta1 subunit that was scarce in endothelial cells of tunica intima (Supplemental Fig. 1; Supplemental Movie 1) (Brenner et al., 2000b). In contrast, immunostaining against endothelial cell marker platelet endothelial cell adhesion molecule-1 (CD-31) showed an opposite pattern of fluorescence intensity that reached its peak within the presumed tunica intima layer, with scarce (if any) staining in tunica media layers (Supplemental Fig. 1; Supplemental Movie 2) (Miettinen et al., 1994). Nuclei and cytoplasm of BK beta1-positive cells were in a perpendicular orientation (Supplemental Movie 1), as opposed to CD-31-positive cells that were oriented parallel to the artery side (Supplemental Movie 2). Perpendicular orientation of the cells relative to the artery side was used to identify smooth muscle cells in filipin staining studies.

We observed increases in filipin-associated fluorescence of the cerebral artery vasculature as CLR concentrations in the incubation media increased (Fig. 1), with a maximum at $\geq 1 \mathrm{mM}$ CLR. CLR enrichment with $0.625 \mathrm{mM}$ CLR approximately represented $\mathrm{EC}_{50}$ for artery vasculature CLR enrichment (Fig. 1B), which is comparable to the degree of CLR increase in de-endothelialized cerebral arteries during the course of a high-CLR diet (Bukiya et al., 2014).

After the efficiency of the CLR-enriching procedure was established, another set of arteries was cannulated and pressurized at $60 \mathrm{~mm} \mathrm{Hg}$. Upon development of myogenic tone, mean external diameter of the arteries reached 150.5 $\pm 5.2 \mu \mathrm{m}$. Changes in arterial diameter were monitored upon exposure of each arterial segment to a single concentration of alcohol ranging from 18 to $100 \mathrm{mM}$. Consistent with our previous findings (Liu et al., 2004), ethanol application resulted in a concentration-dependent decrease in cerebral artery diameter in control arteries with naive CLR level (Fig. 2, A and B). Alcohol-induced constriction reached a maximal value of $10 \% \pm$ $1 \%$ at $50 \mathrm{mM}$ alcohol, with artery diameter returning to prealcohol values upon washout. Notably, as alcohol concentration was increased further $(75-100 \mathrm{mM})$, alcohol-induced constriction started to decrease (Fig. 2, A and B). The loss of alcoholinduced constriction at $75-100 \mathrm{mM}$ EtOH could arise from either BK channel desensitization to alcohol or from alcohol targeting other pathways that mediate dilation. To distinguish between these two possibilities, we tested $100 \mathrm{mM} \mathrm{EtOH}$ in the presence of $1 \mu \mathrm{M}$ paxilline, which blocks BK channels (Zhou and Lingle, 2014). Since the alcohol effect was totally blunted in the presence of paxilline (Supplemental Fig. 2) we did not detect any measurable dilation of the arteries. 
A Naïve CLR

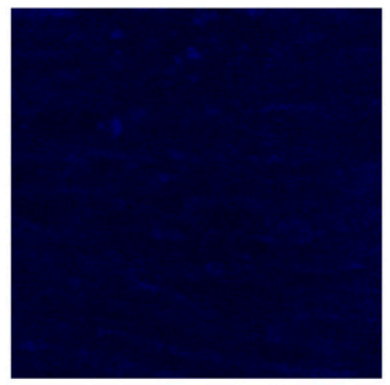

$+0.625 \mathrm{mM}$

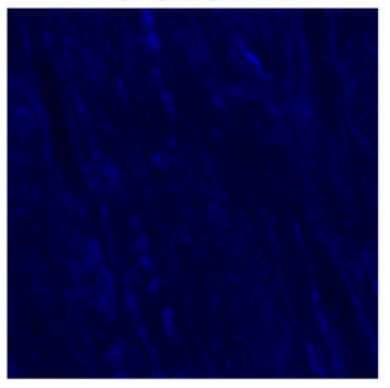

B

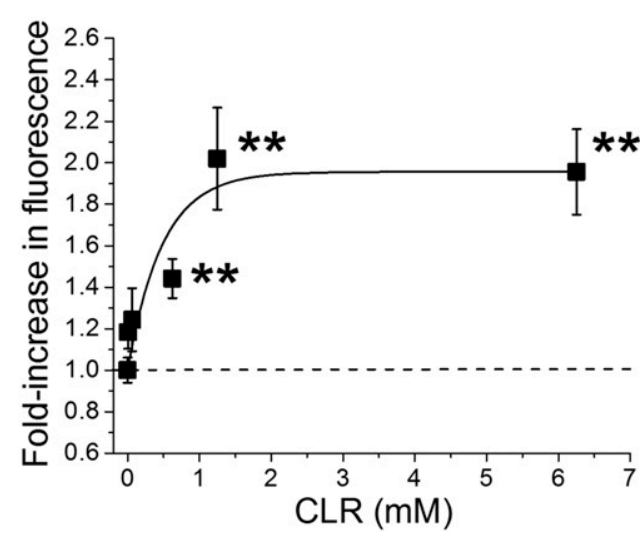

$+0.00625 \mathrm{mM}$

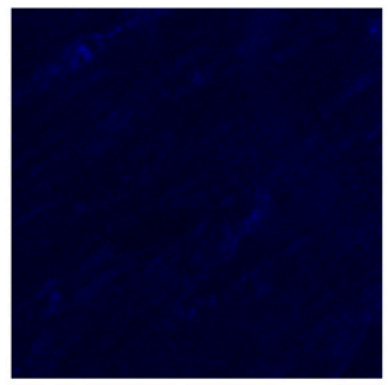

$+1.25 \mathrm{mM}$

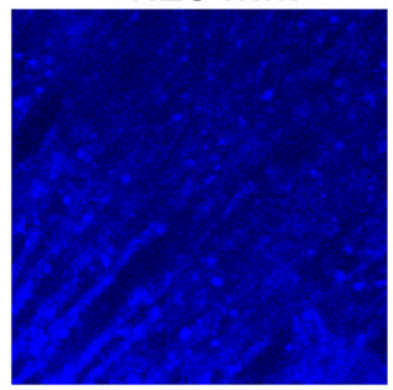

C

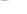
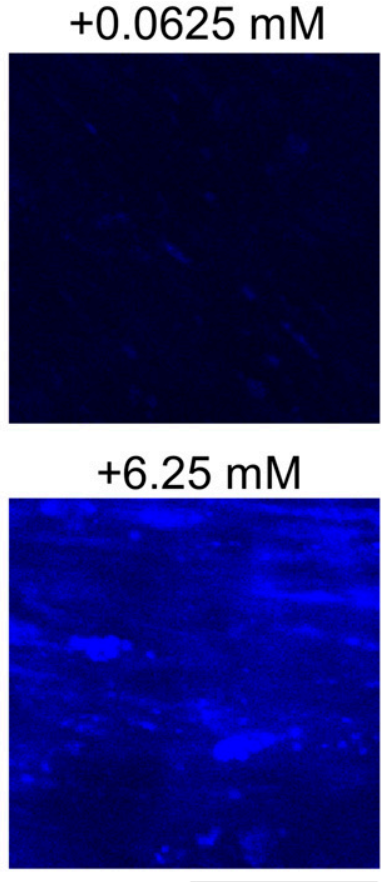

$20 \mu \mathrm{m}$

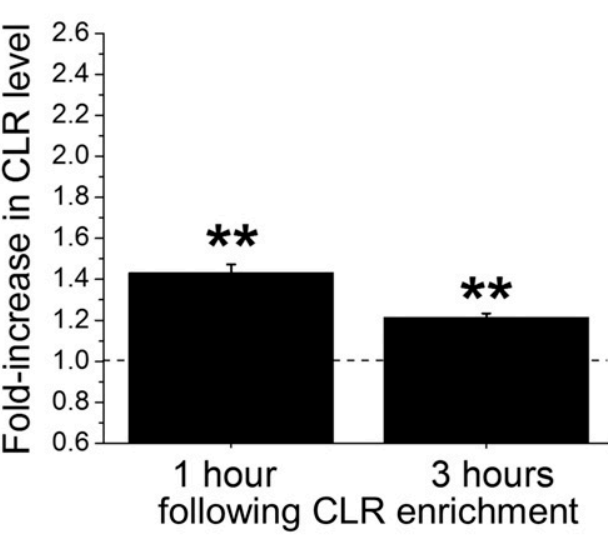

Fig. 1. CLR enrichment of MCAs of rat rendered concentration-dependent increase in VSM CLR content. (A) Original snapshots of filipin-stained segments of rat MCAs following CLR enrichment with various CLR concentrations. (B) Averaged data showing exponential increase in filipin-associated fluorescence as CLR concentration in enriching media was increased. Fluorescence staining experiment was repeated on three independent occasions. Each data point represents averaged data from 8 to 33 artery segments; no more than four artery segments were imaged within each MCA at a given CLR-enriching concentration and each artery was obtained from a separate rat. Data at individual CLR concentrations were compared one by one with data at naive CLR level using the unpaired Student's $t$ test. Data within each sample group passed the Kolmogorov-Smirnov test; thus, these were assumed to follow a Gaussian distribution. ** Denotes different from arteries with naive CLR $(P=$ 0.0033 for $0.625 \mathrm{mM}$ CLR; $P=0.0001$ for $1.25 \mathrm{mM}$ CLR; $P=0.0001$ for $6.25 \mathrm{mM}$ CLR). (C) Averaged fold-increase in CLR level following enrichment with $0.625 \mathrm{mM}$ CLR is sustained over time. ** Denotes different from arteries with naive CLR at corresponding time-points $(P=0.0019$ for 1 hour; $P=0.007$ for 3 hours).
In CLR-enriched arteries, the concentration-response curve was shifted to higher alcohol concentrations. However, the degree of maximal constriction $(8 \% \pm 2 \%$ at $100 \mathrm{mM}$ alcohol $)$ was statistically indistinguishable from that obtained in vessels with naive CLR content (Fig. 2). Thus, at clinically/ toxicologically relevant alcohol concentrations $(<100 \mathrm{mM})$, the alcohol effect was reduced by elevated CLR. The fact that alcohol retained its ability to exert a maximal effect in the presence of CLR, albeit at higher concentration, argues in favor of a competitive interaction between CLR and alcohol to regulate cerebral artery diameter.

The Ability of Coprostanol to Antagonize AlcoholInduced Constriction Rules Out Major BilayerMediated Mechanisms as an Important Force Behind the CLR Protection against Alcohol Effect. Considering that CLR control over the alcohol effect on the BK channel could be observed in the simple protein/lipid environment of artificial lipid bilayers (Crowley et al., 2003), we next used COPR, a CLR derivative that differs drastically from CLR in its ability to modify physical properties of biologic membranes, lipid packing, and condensation in particular (see Discussion).
We performed enrichment of de-endothelialized MCAs of rat with COPR using a protocol identical to that used for CLR (as previously described). Artery enrichment with up to $0.625 \mathrm{mM}$ COPR rendered an amount of steroid content in the smooth muscle layer similar to that evoked by CLR, as detected by filipin staining (Fig. 3, A and B). However, higher amounts of COPR in the enrichment media rendered rapidly declining fluorescence of filipin. The underlying cause of this phenomenon is beyond the scope of the current study.

Enrichment with $0.625 \mathrm{mM}$ COPR rendered a filipinassociated fluorescence signal similar to that of $0.625 \mathrm{mM} \mathrm{CLR}$, and was also associated with protection against alcohol-induced constriction (Fig. 3C). Indeed, alcohol-induced constriction of COPR-enriched arteries did not exceed $1.6 \% \pm 1.0 \%$ (Fig. 3D). This constriction was significantly smaller than alcohol-induced constriction of arteries with naive CLR level $(P=0.0079$ by Mann-Whitney test) and did not differ from alcohol-induced constriction of CLR-enriched arteries (Fig.3D). However, when probed with $100 \mathrm{mM}$ alcohol, constriction of COPR-enriched arteries reached $6.8 \% \pm 1.1 \%$. This constriction was indistinguishable from alcohol-induced constriction of arteries with 
A
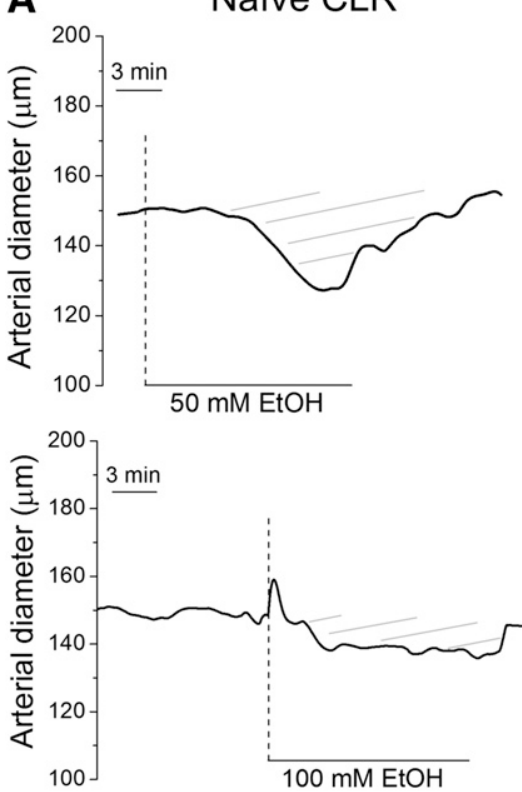

B

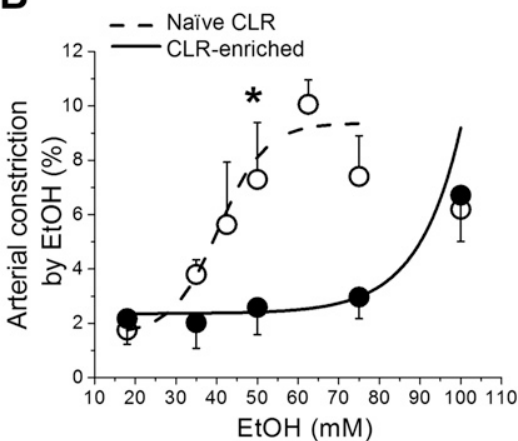

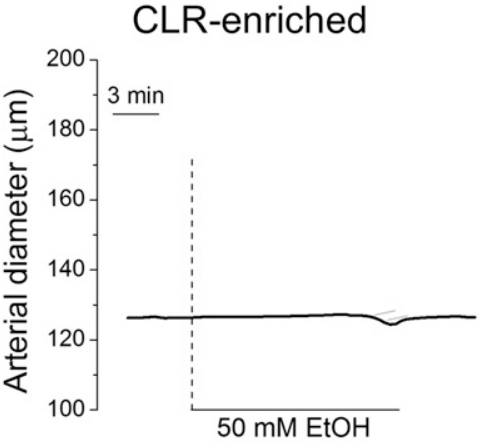
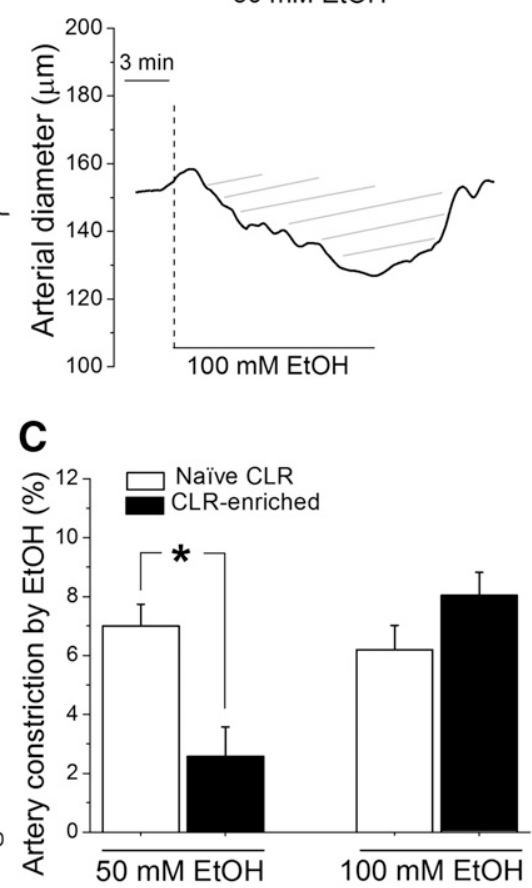

Fig. 2. CLR competed with alcohol over control of constriction in rat de-endothelialized MCAs. (A) Original traces showing that either 50 or $100 \mathrm{mM}$ alcohol (EtOH) reduced arterial dimeter in absence of CLR-modifying treatment, e.g., at naive CLR level (left column of recordings). In contrast, the original trace of CLR-enriched artery (right column of recordings) shows a lack of response to $50 \mathrm{mM}$ alcohol, yet constriction was present when artery was probed with $100 \mathrm{mM}$ alcohol. In all records, vertical dashed lines indicate the start of drug application; hashed shaded areas underscore the degree of artery constriction by alcohol. (B) Concentration-response curve of the averaged decrease in artery diameter in response to alcohol, with and without CLR enrichment in vitro. Each data point represents averaged data from no less than four artery segments, each segment obtained from a separate rat. $*$ Denotes different from arteries with naive CLR, $50 \mathrm{mM} \mathrm{EtOH}(P=0.0263$ by MannWhitney test). (C) Averaged constriction by 50 and $100 \mathrm{mM}$ alcohol with and without CLR enrichment. * Denotes different from arteries with naive CLR, $50 \mathrm{mM}$ EtOH $(P=$ 0.01 by unpaired Student's $t$ test). naive CLR level or CLR-enriched artery segments (Fig. 3E). The overall ability of COPR to influence alcohol-induced constriction in a similar fashion to CLR rules out several bilayer-mediated mechanisms as major determinants of steroid control over alcohol-induced constriction of cerebral arteries. Conceivably, CLR control over alcohol-induced vasoconstriction is mediated by a sterol-sensing protein site(s).

Tyrosine at Position 450 in the BK Channel PoreForming Subunit Mediates the Cholesterol Protection Effect against Alcohol-Induced Constriction of Cerebral Artery. Similar to data obtained from de-endothelialized cerebral arteries of rat (Fig. 2C), arterial constriction by $50 \mathrm{mM}$ alcohol was significantly blunted upon CLR enrichment of mouse MCAs with denuded endothelium (Fig. 4A). While C57BL/6 (WT) mouse arteries with naive CLR level reached on average of $11.1 \% \pm 2.3 \%$ constriction in the presence of $50 \mathrm{mM}$ alcohol, CLR enrichment only led to a $5.3 \% \pm 1.1 \%$ constriction upon alcohol application (Fig. 4A, first pair of bars). However, CLR protection against alcohol was not observed when alcohol was applied at $100 \mathrm{mM}$ (Fig. 4A, right pair of bars).

BK beta1 subunit-lacking mouse arteries with naive CLR have a reduced sensitivity to alcohol (Bukiya et al., 2009a), reaching on average $1.3 \% \pm 0.6 \%$ constriction in the presence of $50 \mathrm{mM}$ alcohol, when compared with their WT counterparts.
However, CLR enrichment fully blunted their remaining residual alcohol sensitivity (Fig. 4B, first pair of bars). In addition, CLR antagonism of alcohol was not observed when EtOH was applied at $100 \mathrm{mM}$ (Fig. 4B, right pair of bars), as found in WT. Thus, the overall pattern of CLR-alcohol interaction on diameter was similar in WT and KCNMB1 KO mice.

To establish the contribution of specific CLR-sensing amino acids to CLR protection against alcohol-induced vasoconstriction, we turned our attention to Tyr450. This amino acid is located in the CRAC4 identified in the slo1 protein cytosolic tail domain, and represents one of the critical CLR-sensing areas on BK slo1. Tyrosine 450 substitution to phenylalanine partially decreased the CLR sensitivity of slo1 cloned from rat cerebral artery myocytes (cbv1) (Singh et al., 2012). To introduce Y450F cbv1 into cerebral arteries, we used KCNMA1 global KO mouse (Meredith et al., 2004). KCNMA1 KO MCAs were permeabilized to introduce pcDNA3 vector carrying the Y450F cbv1-coding nucleotide sequence. Following 3 days of incubation to allow protein expression, BK channel function was verified by electrophysiological means (patch-clamp, Supplemental Fig. 3). BK channel-expressing arteries were de-endothelialized, in vitro pressurized at $60 \mathrm{~mm} \mathrm{Hg}$, and probed with high-KCl solution $(60 \mathrm{mM})$. KCl-induced constriction of Y450F cbv1-containing arteries reached on average $18.3 \%$ $\pm 8.9 \%$, which was similar to the KCl-evoked constriction of 
A
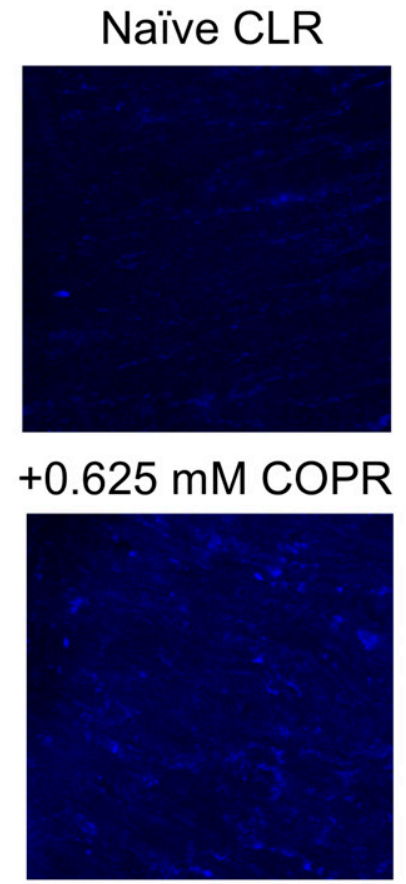

B

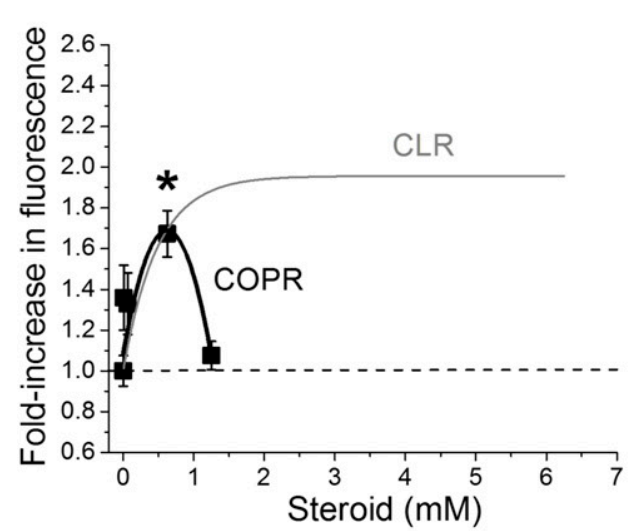

\section{C ${ }^{240}$ COPR-enriched}

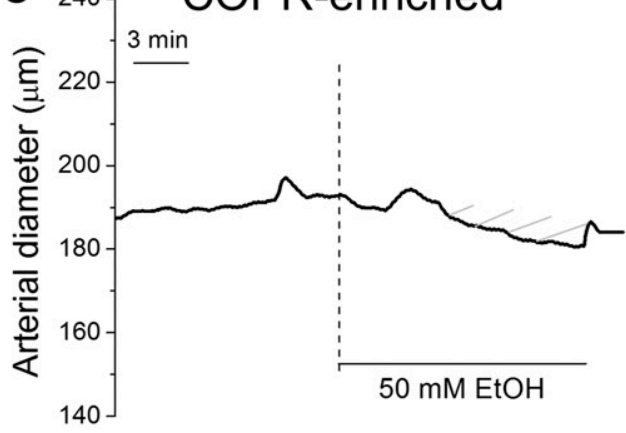

D

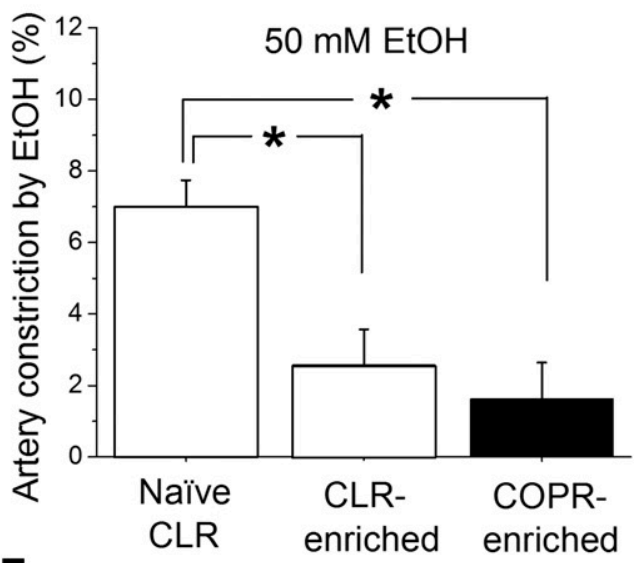

E

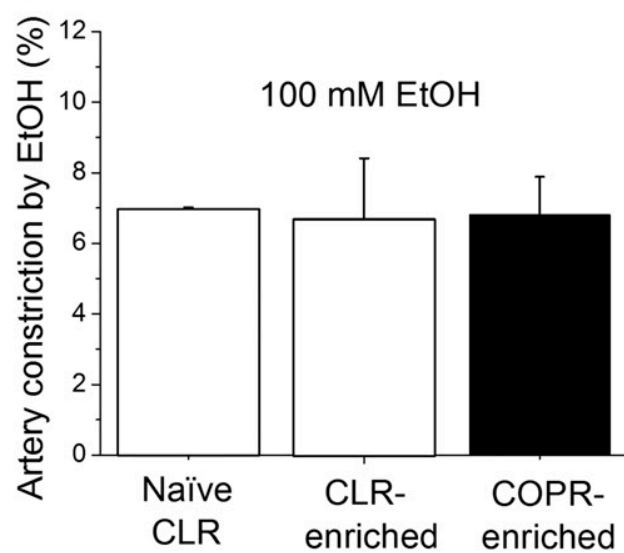

Fig. 3. Enrichment of rat cerebral arteries with COPR rendered protection against alcohol-induced constriction. (A) Original snapshots of filipin-stained segments of rat MCAs with naive CLR (top) and following enrichment with $0.625 \mathrm{mM}$ COPR (bottom). (B) Averaged data showing increase in filipin-associated fluorescence as COPR concentration in enriching media was increased up to $0.625 \mathrm{mM}$. For comparison, the gray curve represents results of artery enrichment with CLR. Fluorescence-staining experiment with COPR was repeated on three independent occasions. Each data point represents averaged data from 5 to 10 artery segments; each artery was obtained from a separate rat. Data at individual COPR concentrations were compared one by one with data at naive CLR level via Mann-Whitney test. * Denotes different from arteries with naive CLR $(P=$ 0.028). (C) Original trace showing COPR protection against alcohol-induced constriction of de-endothelialized MCA of rat. Vertical dashed lines indicate the start of drug application; hashed shaded area underscores limited sensitivity of COPR-enriched artery to alcohol. (D) Averaged constriction by $50 \mathrm{mM}$ alcohol in arteries with naive CLR $(n=28)$, following CLR $(n=4)$ and COPR $(n=5)$ enrichment. * Denotes different from arteries with naive CLR $(P=0.0027$ for analysis of all three groups by Kruskal-Wallis followed by Dunn's post-test). (E) Averaged constriction by $100 \mathrm{mM}$ alcohol in arteries with naive CLR $(n=4)$, following $\operatorname{CLR}(n=$ $7)$ and COPR $(n=5)$ enrichment. arteries permeabilized with WT cbv1 (12.1\% $\pm 3.0 \%$, Fig. 5A). Both outcomes are significantly smaller than KCl-induced constriction of KCNMA1 KO arteries permeabilized with pcDNA3 vector without the cbv1-coding nucleotide sequence $(37.3 \% \pm 4.1 \%$, Fig. $5 \mathrm{~A})$. This reduced vasoconstrictive response to $\mathrm{KCl}$ in cbv1-permeabilized arteries when compared with KCNMA1 $\mathrm{KO}$ is consistent with the primary function of $\mathrm{BK}$ channel in VSM, i.e., its function is to provide negative feedback on myocyte depolarization and contraction (Orio et al., 2002).

Cbv1-permeabilized MCAs were able to retain alcohol-induced constriction, which reached on average $7.6 \% \pm 1.8 \%$ in the presence of $50 \mathrm{mM}$ alcohol (Fig. 5D). As found for rats (as shown previously), constriction by $50 \mathrm{mM}$ alcohol was significantly blunted upon CLR enrichment, only reaching $0.1 \% \pm 1.3 \%$ (Fig. 5D). On one hand, Y450F cbv1-permeabilized arteries constricted in the presence of $50 \mathrm{mM}$ alcohol, up to $11.7 \% \pm 3.2 \%$ (Fig. 5, B and D), underscoring that Y450 was not crucial for alcohol-induced vascular constriction. On the other hand, Y450F cbv1-permeabilized arteries were not protected by CLR against alcohol-induced constriction, which reached $11.2 \% \pm 4.3 \%$ (Fig. 5, C and D). Therefore, Y450 was sufficient for CLR to antagonize alcohol-induced constriction of cerebral arteries.

Remarkably, Y450F cbv1-permeabilized arteries were still protected by COPR enrichment against alcohol-induced constriction, which only reached $1.5 \% \pm 0.6 \%$ (Fig. $6 \mathrm{~A}$ ). Computational docking on COPR onto CRAC4-forming amino acids V444, Y450, and K453 revealed lack of proper COPR orientation along the axis of CRAC4 when compared with CLR docking (Fig. 6B).

\section{Discussion}

In the present work, we identified for the first time the competitive nature of CLR antagonism of alcohol-induced 

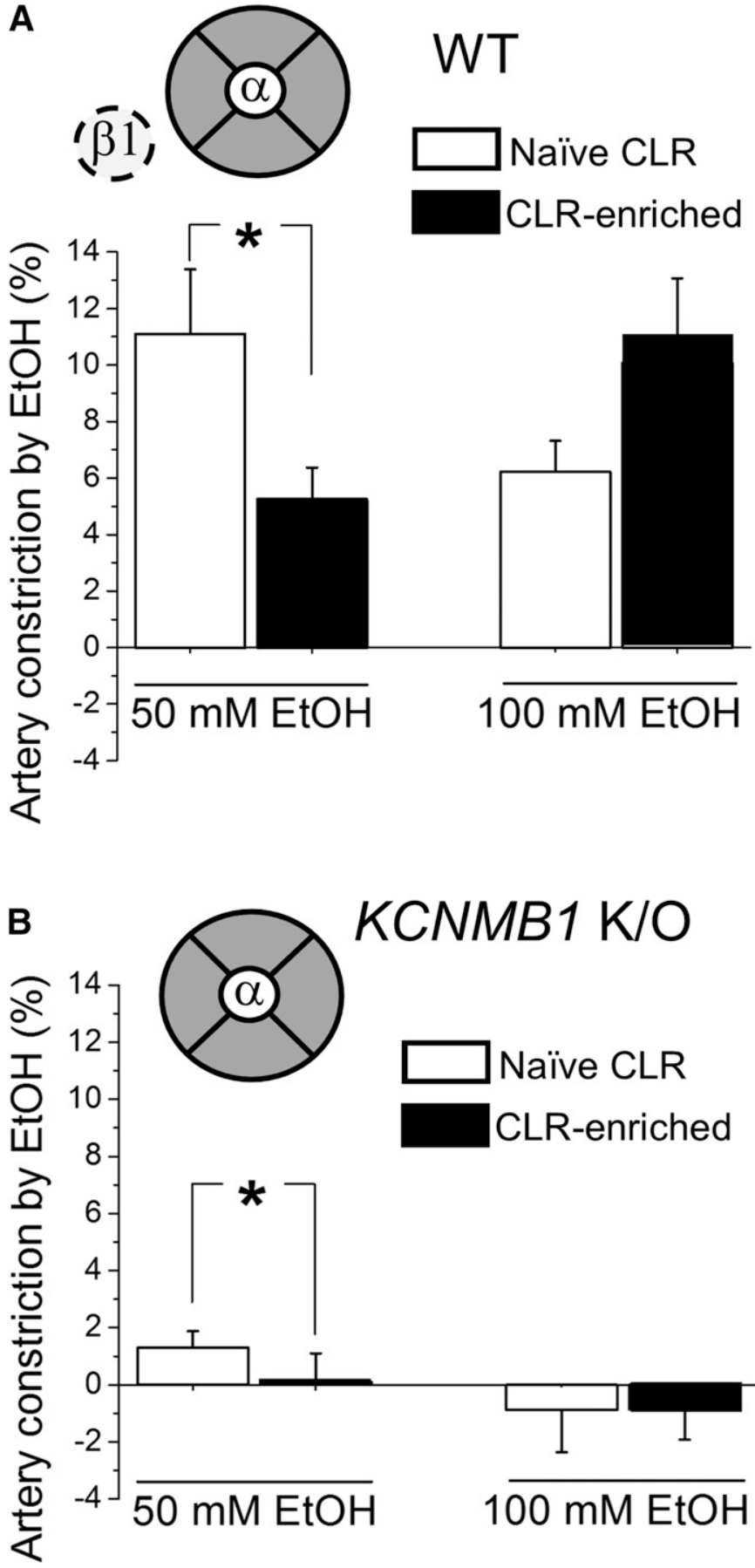

Fig. 4. Pattern of alcohol sensitivity in WT and KCNMB1 KO (BK beta1 subunit-lacking) mouse arteries matched one of the rat MCAs. (A) Averaged constriction by $50 \mathrm{mM} \mathrm{EtOH}$ and $100 \mathrm{mM}$ alcohol with and without CLR enrichment of WT mouse (C57BL/6). The inset depicts the schematic structure of the VSM BK channel consisting of a tetramer of pore-forming alpha subunits accompanied by an accessory beta 1 (view from top). For $50 \mathrm{mM}$ alcohol, $n=6$ in the group with naive CLR and $n=11$ in the CLR-enriched group. For $100 \mathrm{mM}$ alcohol, $n=4$ in each group. Each measurement was performed from a separate artery; arteries within each experimental group were dissected out from different animals. * Denotes different from arteries with naive CLR, $50 \mathrm{mM}$ alcohol $(P=0.02$ by unpaired Student's $t$ test). (B) Averaged constriction by $50 \mathrm{mM}$ alcohol and $100 \mathrm{mM}$ alcohol with and without CLR enrichment of KCNMB1 KO (BK beta1 subunit-lacking) mouse on C57BL/6 background. For $50 \mathrm{mM}$ alcohol, $n=9$ in the group with naive CLR and $n=4$ in the CLR-enriched group. For $100 \mathrm{mM}$ alcohol, $n=4$ in each group. Each measurement was performed from a separate artery; arteries within each experimental group were dissected out from different animals. * Denotes different from arteries with naive CLR, $50 \mathrm{mM}$ alcohol ( $P=0.048$ by Mann-Whitney test). vasoconstriction and a single slo1 residue that mediates this action. Tyrosine 450 in the BK slo1 protein cytosolic tail domain is essential for CLR to protect against alcohol-induced constriction of the cerebral artery.

Changes in blood and/or organ CLR levels are observed during physiologic adaptations and pathophysiological conditions. The former include seasonal and developmental adaptability. For instance, serum CLR levels in healthy humans peak during winter months (Ockene et al., 2004). On the other hand, total blood CLR level increases with age in young or middle-aged adults (Heiss et al., 1980), yet tends to decrease in individuals over 65 years of age (Wallace and Colsher, 1992). Moreover, blood CLR levels are modulated by numerous exogenous factors, including diet and illicit drug use (Wallace and Colsher, 1992; Ginsberg et al., 1995). It is estimated that one-third of adults in the United States have elevated blood CLR levels that require correction with CLR-lowering medication (Schultz et al., 2018). The increased circulating CLR may result in CLR accumulation in various organs, including CLR deposition into the smooth muscle layer of cerebral arteries (Bukiya et al., 2014; Simakova et al., 2017).

Alterations in cerebral artery function have been increasingly recognized as critical contributors to prevalent cerebrovascular and neurologic conditions, including strokes, cerebral vasospasm, migraines, seizures, and dementia (Lee, 1995; Asghar et al., 2011; Washington et al., 2011; Bukiya and Dopico, 2018; Vinters et al., 2018). In the present work, we focused on the CLRalcohol interaction(s) that influenced MCA diameter. MCAs are resistance-size vessels that control blood circulation in many parts of the lateral cerebral cortex (Lee, 1995). Arteries were pressurized in vitro to allow evaluation of organ function at physiologic media and relevant intralumenal pressure. This approach has been widely used for studies with direct clinical implications (González et al., 1999; Aukes et al., 2007; Fike et al., 2012). With regards to alcohol research, several important findings first obtained in pressurized vessels were successfully validated in vivo (Bukiya et al., 2014, Chang et al., 2016). Thus, our current results are likely to provide mechanistic explanations to the earlier observation that dietary CLR protected cerebral arteries from alcohol-induced constriction in vivo via CLR buildup within VSM (Bukiya et al., 2014). Notably, in the human population, males exceed females in excessive drinking (Wilsnack et al., 2009). The current work was performed on male animals, focusing on the gender/sex that is the most affected by excessive alcohol drinking.

Consistent with a previous report (Liu et al., 2004), alcoholinduced constriction of cerebral arteries was concentration dependent (Fig. 2B). Notably, as alcohol concentrations were increased to 75-100 mM, alcohol-induced constriction of arteries with naive CLR started to decline compared with constriction evoked by $35-50 \mathrm{mM}$ alcohol. This was likely due to the desensitization of ethanol's targets in MCA. Indeed, we did not detect dilatory responses when the artery was treated with $100 \mathrm{mM}$ EtOH in the presence of the BK channel blocker paxilline (Supplemental Fig. 2). Thus, loss of the alcohol effect at high EtOH levels was due to the targeting of BK channels. Desensitization of receptor and ion channel function by ethyl alcohol has been widely reported, including tolerance to protracted high-dose alcohol challenge in BK channels (Moranta et al., 2006; Dopico and Lovinger, 2009).

When compared with lower alcohol levels, constriction in the presence of $100 \mathrm{mM}$ EtOH was characterized by a brief 

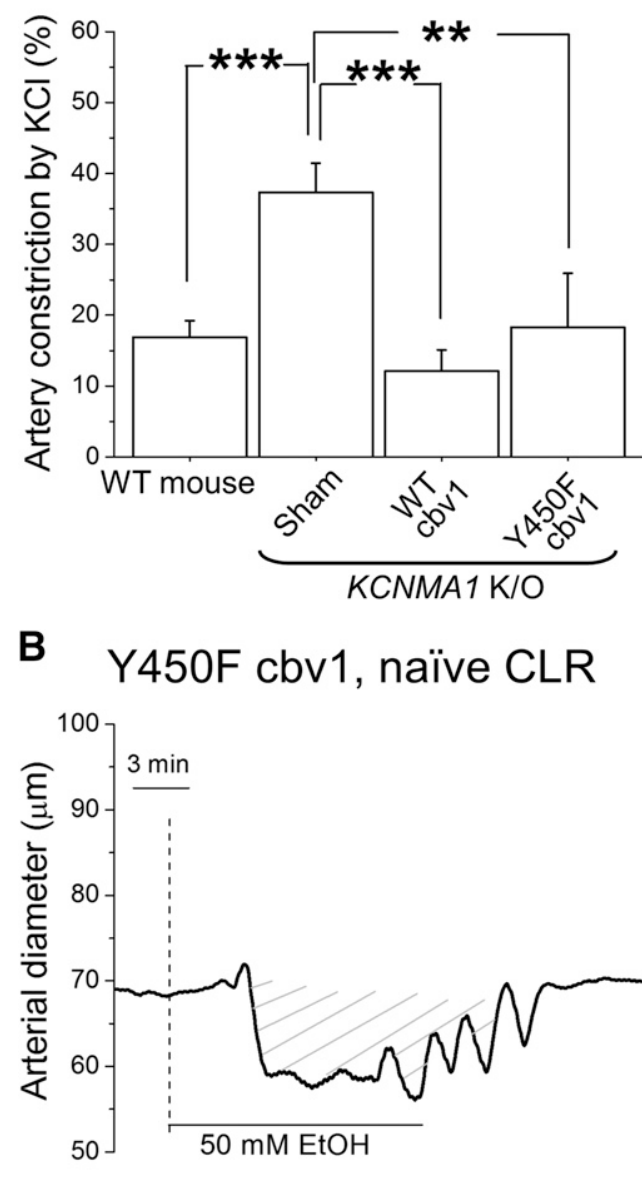

C Y450F cbv1, CLR-enriched

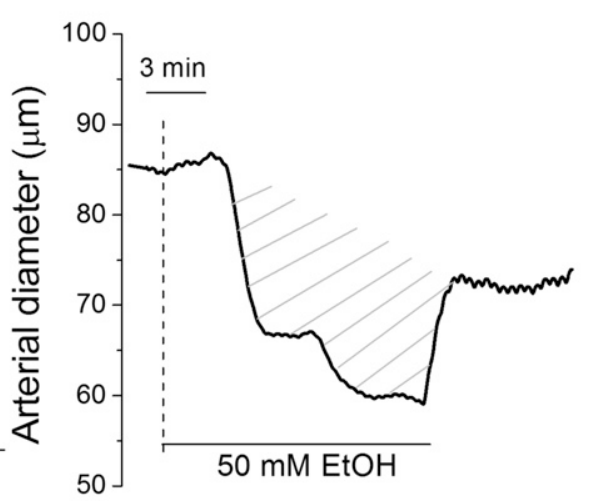

D

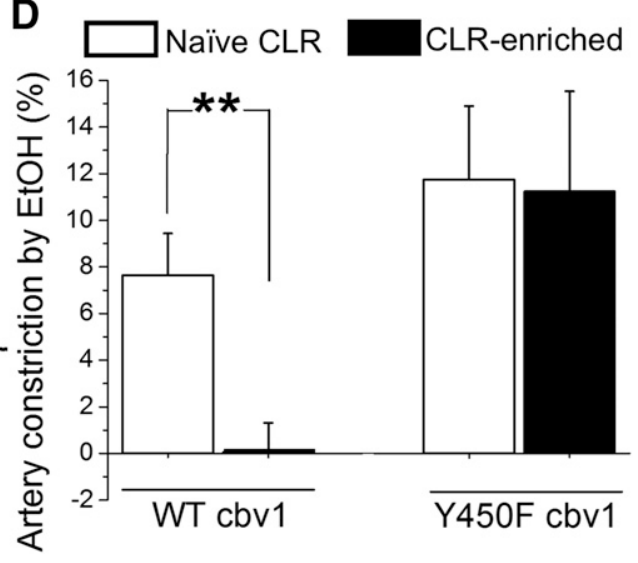

Fig. 5. Mutation of CLR-sensing site at Y450 within BK pore-forming (KCNMA1) subunit fully ablated CLR protection against alcohol-induced vasoconstriction in mouse cerebral artery. (A) Averaged data showing MCA constriction by $60 \mathrm{mM}$ $\mathrm{KCl}$ in freshly dissected MCAs of WT mouse ( $n=16$ ), and following KCNMA1 KO mouse artery permeabilization and introduction of pcDNA3 (negative control, sham, $n=9$ ), pcDNA3-WT cbv1 (positive control, $n=11$ ), and pcDNA3-Y450F cbv1 $(n=4)$. *** Denotes different from sham $(P<0.001) ; * * P<0.01$. Statistical significance was established using one-way analysis of variance with Tukey's post-test. (B) Original trace showing alcohol-induced constriction of de-endothelialized MCA from KCNMA1 KO mouse on C57BL/6 background following permeabilization and introduction of Y450F cbv1. Here and in (C), vertical dashed lines indicate the start of drug application; hashed shaded area underscores effect of $50 \mathrm{mM}$ alcohol on artery diameter. (C) Original trace showing lack of blunting of alcohol-induced constriction in de-endothelialized Y450F cbv1permeabilized MCA from KCNMA1 KO mouse following CLR enrichment. (D) Averaged constriction by $50 \mathrm{mM}$ alcohol with and without CLR enrichment of KCNMA1 KO mouse MCAs following permeabilization with either WT cbv1 (first pair of bars) or Y450F cbv1 (second pair of bars). For WT cbv1, naive and CLRenriched groups contained seven arteries each. ** Denotes different from naive CLR $(P=0.0047$ by unpaired $t$ test). For Y450F cbv1, the naive CLR group contained six arteries, while the CLR-enriched group contained five arteries. $P=0.6623$ by Mann-Whitney test. vasodilatory spike at the beginning of alcohol administration into the artery perfusion chamber (Fig. 2A, bottom traces). Such a spike may represent a transient effect on an unidentified molecular target. Considering that the spike in question is brief compared with the long-lasting constrictive effect, and that $100 \mathrm{mM}$ EtOH is at the limit of physiologically relevant alcohol concentrations (Heatley and Crane, 1990), mechanistic studies regarding this phenomenon were omitted from the present work.

While CLR enrichment protected against the vasoconstriction evoked by a wide range of alcohol concentrations (18-75 mM), CLR failed to alter the efficacy of alcohol. Indeed, when alcohol concentration was raised to $100 \mathrm{mM}$, alcohol-induced constriction was indistinguishable between arteries with naive CLR and following CLR enrichment. This outcome is consistent with a competitive interaction between two ligands for a common target and/or mechanism (molecular site of interaction or signaling pathway) (Zuideveld et al., 2002).

The major effector of CLR and alcohol in cerebral artery smooth muscle, i.e., the BK channel, tends to cluster in CLRrich, rather thick lipid raft membrane domains (reviewed by Dopico et al., 2012). Lipid raft disruption by manipulations with cholesterol level may affect BK channel function, and possibly its response to alcohol at the channel and organ level. Indeed, previous studies demonstrated that altering the thickness of the bilayer by adjusting the acyl chain length of the component lipids affected the time course of the acute response to alcohol, and could transform alcohol-induced channel potentiation to inhibition (reviewed in Treistman and Martin (2009)). However, alcohol-cholesterol interactions can be observed in rather isolated systems-artificial lipid membranes that lack the complex protein and lipid content of native membranes (Crowley et al., 2003; Bukiya et al., 2011). Thus, lipid raft-specific proteins are not needed for cholesterol-alcohol interactions in the control of BK channel function.

Previous data documented that CLR protection against alcohol-induced constriction could be observed in KCNMB1 KO mouse arteries (Bisen et al., 2016). Moreover, CLR antagonism of alcohol action on BK channels was documented in a simple system conformed by slo1 channel proteins and one or two phospholipid species bilayers (Crowley et al., 2003). The latter finding raised the question of whether bilayer-mediated mechanisms, rather than ligand interactions with slo1 proteins, are involved in CLR-alcohol interaction. Reciprocal actions on time-dwell distributions led to speculation that CLR and alcohol functionally antagonized each other via opposing actions on bilayer properties (Crowley et al., 2003). Indeed, CLR diminishes the lipid/membrane partition coefficient of a variety of small anesthetics, such as halothane (Lechleiter et al., 1986), uncharged pentobarbitone (Miller and $\mathrm{Yu}, 1977$ ), and benzyl alcohol (Colley and Metcalfe, 1972). 


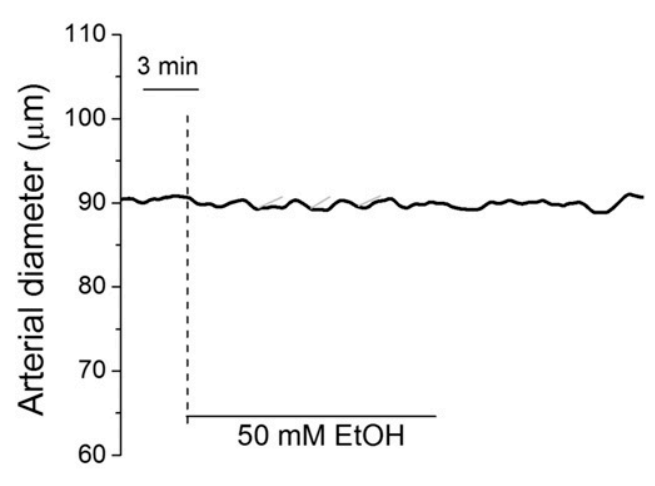

B

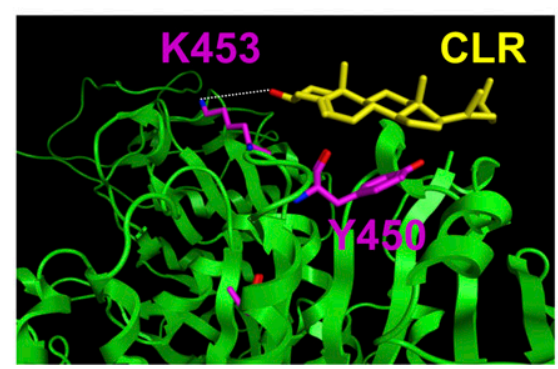

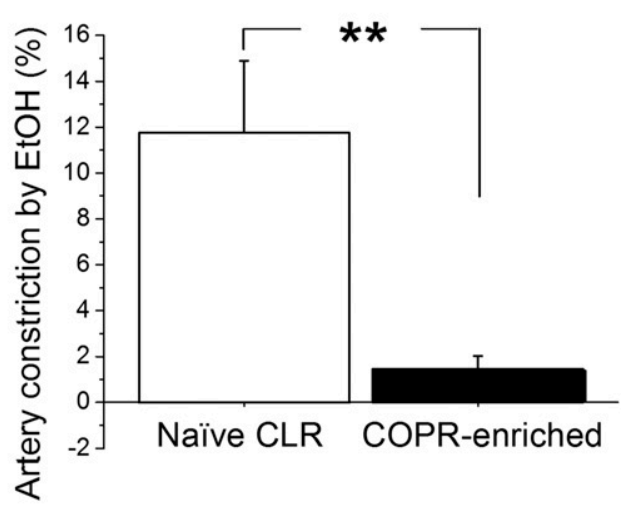

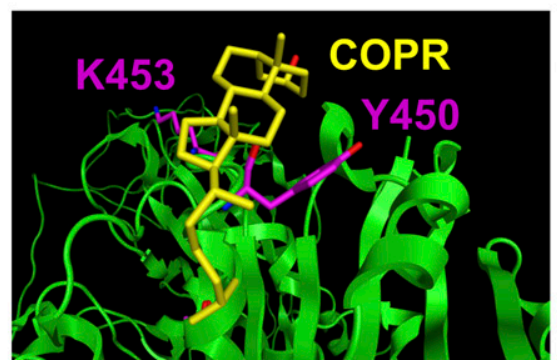

Fig. 6. Mutation of CLR-sensing site at Y450 within BK pore-forming (KCNMA1) subunit did not ablate COPR protection against alcohol-induced vasoconstriction in mouse cerebral artery. (A) Original trace (left) and averaged data (right) showing lack of alcohol-induced constriction of de-endothelialized MCA from KCNMA1 KO mouse on C57BL/6 background loaded with Y450F cbv1 and subjected to COPR enrichment. Naive CLR and COPR-enriched groups contained seven and eight arteries, respectively. ** Denotes different from naive CLR ( $P=0.004$ by Mann-Whitney test). (B) Computational docking onto CRACR4-forming amino acids revealed differential docking modes for CLR (left) vs. COPR (right) molecules. Light dotted line depicts presumed hydrogen bonding between CLR hydroxyl group and K453 (Singh et al., 2012).
Thus, it is expected that CLR enrichment would make it difficult for alcohol to partition into the membrane and reach the effective concentrations that would evoke vasoconstriction. In a previous study, we demonstrated that enantiomeric CLR also protected against alcohol-induced constriction of cerebral arteries (Bisen et al., 2016). Enantiomeric CLR is a mirror image of the CLR molecule and is expected to modify bulk physical properties of the membrane similar to CLR (Mannock et al., 2003). Collectively, all of these previous findings seem to favor the idea that the primary mechanism(s) mediating CLRalcohol interaction with BK channels, and eventually artery contraction, resides within membrane bilayer properties. However, in the present work we used the enrichment of cerebral arteries with COPR (Fig. 3). Unlike CLR, COPR has all saturated carbon-carbon bonds in its steroid nucleus and a cis fusion of rings $\mathrm{A}$ and $\mathrm{B}$, which orients its hydroxyl group in C3 toward the alpha-face of the steroid molecule (Pine, 1987). Given this chemical structure, COPR exerts membrane bilayer effects that are opposite to or significantly smaller than the effects of CLR. For instance, while CLR was shown to promote dipalmitoylphosphatidylcholine domain formation, COPR strongly inhibited this effect, acting as anti-CLR (Xu and London, 2000). In another study, COPR evoked a much lesser effect on the Stokes shift of the voltagesensitive styrylpyridinium probe di-8-ANEPPS and the orientational polarizability of the unilamellar phosphatidylcholine vesicles compared with CLR (Le Goff et al., 2007). However, the similar ability of COPR and CLR to protect against alcohol-induced constriction (Fig. 3) rules out alterations in membrane physical properties (including alteration of lipid raft physical characteristics) as the primary mechanism of CLR-alcohol interaction. Rather, CLR protection against the alcohol constrictive effect is linked to the presence of steroid-sensing site(s) in the slo1 protein.
We have previously identified seven CRAC motifs (CRACs 4-10) within the cytosolic tail domain of slo1 that influence the response of slo1 channels to this lipid (Singh et al., 2012). Our current study shows that the slo1 Y450F substitution removes CLR protection against alcohol-induced vasoconstriction (Fig. 5). This conclusion is consistent with previous observations (Crowley et al., 2003; Yuan et al., 2011; Bisen et al., 2016) and our own current data (Fig. 4), suggesting a critical role of the BK slo1 subunit in enabling CLR control over its alcohol effect. Tyrosine 450 is a central amino acid located within CRAC4 of the BK slo1 (cbv1) (Singh et al., 2012), and the Y450F substitution partially decreases slo1 sensitivity to $33 \mathrm{~mol} \% \mathrm{CLR}$ in planar lipid bilayers (Singh et al., 2012). However, in our current work Y450F fully ablated the ability of CLR enrichment to control alcoholinduced vasoconstriction. Considering that mammalian cellular membranes contain between 20 and 50 mol\% CLR (van Meer et al., 2008), it is likely that in cerebral artery myocyte membranes CLR concentration exceeds 33 mol\%. Therefore, the differential ability of Y450 to control CLR sensitivity of the channel versus alcohol-induced constriction may be explained by the low affinity of CRAC4 to CLR. In addition, the complexity of the cellular environment in the cerebral artery compared with artificial lipid membranes may provide Y450 with more significant control over the alcohol effect on artery diameter.

The truncation of slo1 downstream CRAC4 renders a channel that is lacking in CRACs 5-10. Interestingly, the Y450F substitution in this truncated slo1 was fully efficient in preventing CLR to modulate channel activity (Singh et al., 2012). This finding raised the speculation of a competitive interaction between the several CRACs in slo1 proteins for CLR sensitivity (Singh et al., 2012). The possibility that more than one CRAC or steroid-sensing region could contribute to 
shaping the alcohol effect is further supported by our data showing the persistence of COPR protection against alcoholinduced constriction even in the presence of Y450F cbv1 mutant (Fig. 6). Whether CRAC motifs other than CRAC4 contribute to the competitive interaction between CLR and alcohol in control of cerebral artery diameter, and whether these CRAC motifs represent true binding sites for steroids, remains to be tested experimentally.

In conclusion, our data unveil the competitive nature of CLR interaction with alcohol in controlling cerebral artery diameter, and document the critical role of a single amino acid (Y450) within the BK channel pore-forming subunit in enabling CLR control over the alcohol constrictive effect at the organ level.

\section{Acknowledgments}

We deeply thank Dr. Andrea Meredith (University of Maryland) for providing KCNMA1 KO mice. We also deeply thank Dr. Jonathan Jaggar and laboratory member Dr. Dennis Leo (University of Tennessee Health Science Center) for help with the artery permeabilization procedure. The authors deeply thank Dr. Richard Redfearn (Office of Research, University of Tennessee Health Science Center) for copyediting the manuscript.

\section{Authorship Contributions}

Participated in research design: North, Bisen, Dopico, Bukiya.

Conducted experiments: North, Bisen, Bukiya.

Performed data analyses: North, Bisen, Dopico, Bukiya.

Wrote or contributed to the writing of the manuscript: North, Bisen, Dopico, Bukiya.

\section{References}

Altura BM and Altura BT (1984) Alcohol, the cerebral circulation and strokes Alcohol 1:325-331.

Asghar MS, Hansen AE, Amin FM, van der Geest RJ, Koning Pv, Larsson HB, Olesen $\mathrm{J}$, and Ashina M (2011) Evidence for a vascular factor in migraine. Ann Neurol 69: $635-645$.

Aukes AM, Vitullo L, Zeeman GG, and Cipolla MJ (2007) Pregnancy prevents hypertensive remodeling and decreases myogenic reactivity in posterior cerebra arteries from Dahl salt-sensitive rats: a role in eclampsia? Am J Physiol Heart Circ Physiol 292:H1071-H1076.

Bisen S, Seleverstov O, Belani J, Rychnovsky S, Dopico AM, and Bukiya AN (2016) Distinct mechanisms underlying cholesterol protection against alcohol-induced BK channel inhibition and resulting vasoconstriction. Biochim Biophys Acta 1861: 1756-1766.

Brenner R, Jegla TJ, Wickenden A, Liu Y, and Aldrich RW (2000a) Cloning and functional characterization of novel large conductance calcium-activated potassium channel beta subunits, hKCNMB3 and hKCNMB4. J Biol Chem 275:6453-6461.

Brenner R, Peréz GJ, Bonev AD, Eckman DM, Kosek JC, Wiler SW, Patterson AJ, Nelson MT, and Aldrich RW (2000b) Vasoregulation by the beta1 subunit of the calcium-activated potassium channel. Nature 407:870-876.

Bukiya A, Dopico AM, Leffler CW, and Fedinec A (2014) Dietary cholesterol protects against alcohol-induced cerebral artery constriction. Alcohol Clin Exp Res 38: $1216-1226$.

Bukiya AN and Dopico AM (2018) Fetal cerebral circulation as target of maternal alcohol consumption. Alcohol Clin Exp Res 42:1006-1018.

Bukiya AN, Liu J, and Dopico AM (2009a) The BK channel accessory beta1 subunit determines alcohol-induced cerebrovascular constriction. FEBS Lett $\mathbf{5 8 3}$ 2779-2784.

Bukiya AN, Liu J, Toro L, and Dopico AM (2007) Beta1 (KCNMB1) subunits mediate lithocholate activation of large-conductance $\mathrm{Ca}^{2+}$-activated $\mathrm{K}^{+}$channels and dilation in small, resistance-size arteries. Mol Pharmacol 72:359-369.

Bukiya AN, McMillan JE, Fedinec AL, Patil SA, Miller DD, Leffler CW, Parrill AL, and Dopico AM (2013) Cerebrovascular dilation via selective targeting of the cholane steroid-recognition site in the BK channel $\beta 1$-subunit by a novel nonsteroidal agent. Mol Pharmacol 83:1030-1044.

Bukiya AN, Vaithianathan T, Kuntamallappanavar G, Asuncion-Chin M, and Dopico AM (2011) Smooth muscle cholesterol enables BK $\beta 1$ subunit-mediated channel inhibition and subsequent vasoconstriction evoked by alcohol. Arterioscler Thromb Vasc Biol 31:2410-2423.

Bukiya AN, Vaithianathan T, Toro L, and Dopico AM (2009b) Channel $\beta 2-4$ subunits fail to substitute for $\beta 1$ in sensitizing BK channels to lithocholate. Biochem Biophys Res Commun 390:995-1000.

Chang J, Fedinec AL, Kuntamallappanavar G, Leffler CW, Bukiya AN, and Dopico AM (2016) Endothelial nitric oxide mediates caffeine antagonism of alcoholinduced cerebral artery constriction. J Pharmacol Exp Ther 356:106-115.

Colley CM and Metcalfe JC (1972) The localisation of small molecules in lipid bilayers. FEBS Lett 24:241-246.
Crowley JJ, Treistman SN, and Dopico AM (2003) Cholesterol antagonizes ethanol potentiation of human brain $\mathrm{BK}_{\mathrm{Ca}}$ channels reconstituted into phospholipid bilayers. Mol Pharmacol 64:365-372.

Dopico AM, Bukiya AN, and Singh AK (2012) Large conductance, calcium- and voltage-gated potassium (BK) channels: regulation by cholesterol. Pharmacol Ther 135:133-150.

Dopico AM and Lovinger DM (2009) Acute alcohol action and desensitization of ligand-gated ion channels. Pharmacol Rev 61:98-114.

Dopico AM and Tigyi GJ (2007) A glance at the structural and functional diversity of membrane lipids. Methods Mol Biol 400:1-13.

Fike CD, Kaplowitz M, Zhang Y, Dantuma M, and Madden JA (2012) Effect of a phosphodiesterase 5 inhibitor on pulmonary and cerebral arteries of newborn piglets with chronic hypoxia-induced pulmonary hypertension. Neonatology 101 $28-39$

Ginsberg HN, Karmally W, Siddiqui M, Holleran S, Tall AR, Blaner WS, and Ramakrishnan R (1995) Increases in dietary cholesterol are associated with modest increases in both LDL and HDL cholesterol in healthy young women. Arterioscler Thromb Vasc Biol 15:169-178.

González R, Manso AM, and Marín J (1999) Effect of ouabain in pressurized middle cerebral arteries from normotensive and hypertensive rats. Methods Find Exp Clin Pharmacol 21:89-97.

Haldar S, Kanaparthi RK, Samanta A, and Chattopadhyay A (2012) Differential effect of cholesterol and its biosynthetic precursors on membrane dipole potential. Biophys J 102:1561-1569.

Heatley MK and Crane $J$ (1990) The blood alcohol concentration at post-mortem in 175 fatal cases of alcohol intoxication. Med Sci Law 30:101-105.

Heiner AL, Gibbons E, Fairbourn JL, Gonzalez LJ, McLemore CO, Brueseke TJ, Judd AM, and Bell JD (2008) Effects of cholesterol on physical properties of human erythrocyte membranes: impact on susceptibility to hydrolysis by secretory phospholipase $\mathrm{A}_{2}$. Biophys $J$ 94:3084-3093.

Heiss G, Tamir I, Davis CE, Tyroler HA, Rifkand BM, Schonfeld G, Jacobs D, and Frantz ID, Jr (1980) Lipoprotein-cholesterol distributions in selected North American populations: the lipid research clinics program prevalence study. Circulation 61:302-315

Ivanovic B and Tadic M (2015) Hypercholesterolemia and hypertension: two sides of the same coin. Am J Cardiovasc Drugs 15:403-414.

Lechleiter J, Wells M, and Gruener R (1986) Halothane-induced changes in acetylcholine receptor channel kinetics are attenuated by cholesterol. Biochim Biophys Acta 856:640-645.

Lee RM (1995) Morphology of cerebral arteries. Pharmacol Ther 66:149-173.

Le Goff G, Vitha MF, and Clarke RJ (2007) Orientational polarisability of lipid membrane surfaces. Biochim Biophys Acta 1768:562-570.

Lesh RE, Somlyo AP, Owens GK, and Somlyo AV (1995) Reversible permeabilization. A novel technique for the intracellular introduction of antisense oligodeoxynucleotides into intact smooth muscle. Circ Res 77:220-230.

Li H, Horke S, and Förstermann U (2014) Vascular oxidative stress, nitric oxide and atherosclerosis. Atherosclerosis 237:208-219.

Liu P, Xi Q, Ahmed A, Jaggar JH, and Dopico AM (2004) Essential role for smooth muscle BK channels in alcohol-induced cerebrovascular constriction. Proc Nat Acad Sci USA 101:18217-18222.

Mannock DA, McIntosh TJ, Jiang X, Covey DF, and McElhaney RN (2003) Effects of natural and enantiomeric cholesterol on the thermotropic phase behavior and structure of egg sphingomyelin bilayer membranes. Biophys $J$ 84:1038-1046.

Martín MG, Pfrieger F, and Dotti CG (2014) Cholesterol in brain disease: sometimes determinant and frequently implicated. EMBO Rep 15:1036-1052.

Meredith AL, Thorneloe KS, Werner ME, Nelson MT, and Aldrich RW (2004 Overactive bladder and incontinence in the absence of the BK large conductance $\mathrm{Ca}^{2+}$-activated $\mathrm{K}^{+}$channel. J Biol Chem 279:36746-36752.

Miettinen M, Lindenmayer AE, and Chaubal A (1994) Endothelial cell markers CD31, CD34, and BNH9 antibody to $\mathrm{H}$ - and Y-antigens-evaluation of their specificity and sensitivity in the diagnosis of vascular tumors and comparison with von Willebrand factor. Mod Pathol 7:82-90.

Miller KW and Yu SC (1977) The dependence of the lipid bilayer membrane: buffer partition coefficient of pentobarbitone on $\mathrm{pH}$ and lipid composition. $\mathrm{Br} J$ Pharmacol 61:57-63.

Miller WL and Auchus RJ (2011) The molecular biology, biochemistry, and physiology of human steroidogenesis and its disorders. Endocr Rev 32:81-151.

Moranta D, Esteban S, and García-Sevilla JA (2006) Ethanol desensitizes cannabinoid $\mathrm{CB}_{1}$ receptors modulating monoamine synthesis in the rat brain in vivo. Neurosci Lett 392:58-61.

Muller CP, Stephany DA, Winkler DF, Hoeg JM, Demosky S.J Jr, and Wunderlich JR (1984) Filipin as a flow microfluorometry probe for cellular cholesterol. Cytometry 5:42-54

Ockene IS, Chiriboga DE, Stanek EJ, III, Harmatz MG, Nicolosi R, Saperia G, Well AD, Freedson P, Merriam PA, Reed G, et al. (2004) Seasonal variation in serum cholesterol levels: treatment implications and possible mechanisms. Arch Intern Med 164:863-870.

Orio P, Rojas P, Ferreira G, and Latorre R (2002) New disguises for an old channel: MaxiK channel $\beta$-subunits. News Physiol Sci 17:156-161.

Pine HP (1987) Steroids, in Organic Chemistry (Pine HP ed) 5th ed, pp 876, McGrawHill Book Company, New York, p. 876.

Salkoff L, Butler A, Ferreira G, Santi C, and Wei A (2006) High-conductance potassium channels of the SLO family. Nat Rev Neurosci 7:921-931.

Schultz BG, Patten DK, and Berlau DJ (2018) The role of statins in both cognitive impairment and protection against dementia: a tale of two mechanisms. Transl Neurodegener 7:5.

Simakova MN, Bisen S, Dopico AM, and Bukiya AN (2017) Statin therapy exacerbates alcohol-induced constriction of cerebral arteries via modulation of ethanolinduced BK channel inhibition in vascular smooth muscle. Biochem Pharmacol 145:81-93. 
Singh AK, McMillan J, Bukiya AN, Burton B, Parrill AL, and Dopico AM (2012) Multiple cholesterol recognition/interaction amino acid consensus (CRAC) motifs in cytosolic $\mathrm{C}$ tail of Slo1 subunit determine cholesterol sensitivity of $\mathrm{Ca}^{2+}-$ and voltage-gated $\mathrm{K}^{+}$(BK) channels. J Biol Chem 287:20509-20521.

Treistman SN and Martin GE (2009) BK channels: mediators and models for alcohol tolerance. Trends Neurosci 32:629-637.

van Meer G, Voelker DR, and Feigenson GW (2008) Membrane lipids: where they are and how they behave. Nat Rev Mol Cell Biol 9:112-124.

van Rooy MJ and Pretorius E (2014) Obesity, hypertension and hypercholesterolemia as risk factors for atherosclerosis leading to ischemic events. Curr Med Chem $\mathbf{2 1}$ 2121-2129.

Veerkamp JH (1972) Lipids of the cell plasma membrane. Biomembranes 3:159-179.

Vinters HV, Zarow C, Borys E, Whitman JD, Tung S, Ellis WG, Zheng L, and Chui HC (2018) Review: vascular dementia: clinicopathologic and genetic considerations. Neuropathol Appl Neurobiol 44:247-266.

Wallace RB and Colsher PL (1992) Blood lipid distributions in older persons. Prevalence and correlates of hyperlipidemia. Ann Epidemiol 2:15-21.

Washington CW and Zipfel GJ; Participants in the International Multi-disciplinary Consensus Conference on the Critical Care Management of Subarachnoid Hemorrhage (2011) Detection and monitoring of vasospasm and delayed cerebral ischemia: a review and assessment of the literature. Neurocrit Care 15:312-317.

Wilsnack RW, Wilsnack SC, Kristjanson AF, Vogeltanz-Holm ND, and Gmel G (2009) Gender and alcohol consumption: patterns from the multinational GENACIS project. Addiction 104:1487-1500.

Wu N, Zhang X, Jia P, and Jia D (2015) Hypercholesterolemia aggravates myocardial ischemia reperfusion injury via activating endoplasmic reticulum stress-mediated apoptosis. Exp Mol Pathol 99:449-454.

Xi Q, Adebiyi A, Zhao G, Chapman KE, Waters CM, Hassid A, and Jaggar JH (2008) $\mathrm{IP}_{3}$ constricts cerebral arteries via $\mathrm{IP}_{3}$ receptor-mediated TRPC3 channel activation and independently of sarcoplasmic reticulum $\mathrm{Ca}^{2+}$ release. Circ Res 102 : $1118-1126$

$\mathrm{Xu} \mathrm{X}$ and London E (2000) The effect of sterol structure on membrane lipid domains reveals how cholesterol can induce lipid domain formation. Biochemistry 39: 843-849.

Yuan C, Chen M, Covey DF, Johnston LJ, and Treistman SN (2011) Cholesterol tuning of BK ethanol response is enantioselective, and is a function of accompanying lipids. PLoS One 6:e27572.

Zakhari S (1997) Alcohol and the cardiovascular system: molecular mechanisms for beneficial and harmful action. Alcohol Health Res World 21:21-29.

Zhang C, Qin YY, Chen Q, Jiang H, Chen XZ, Xu CL, Mao PJ, He J, and Zhou YH (2014) Alcohol intake and risk of stroke: a dose-response meta-analysis of prospective studies. Int $J$ Cardiol 174:669-677.

Zhou Y and Lingle CJ (2014) Paxilline inhibits BK channels by an almost exclusively closed-channel block mechanism. J Gen Physiol 144:415-440.

Zidovetzki R and Levitan I (2007) Use of cyclodextrins to manipulate plasma membrane cholesterol content: evidence, misconceptions and control strategies. Biochim Biophys Acta 1768:1311-1324.

Zuideveld KP, Treijtel N, Maas HJ, Gubbens-Stibbe JM, Peletier LA, van Der Graaf PH, and Danhof M (2002) A competitive interaction model predicts the effect of WAY-100,635 on the time course of R-(+)-8-hydroxy-2-(di- $n$-propylamino)tetralininduced hypothermia. J Pharmacol Exp Ther 300:330-338.

Address correspondence to: Anna N. Bukiya, Department of Pharmacology, University of Tennessee Health Science Center, 71 S. Manassas Street, Room \#205, Memphis, TN 38103. E-mail: abukiya@uthsc.edu 